\title{
Comments Regarding "On the Performance/Complexity Tradeoff in Block Turbo Decoder Design"
}

\author{
Philippa A. Martin, Senior Member, IEEE, and Desmond P. Taylor, Life Fellow, IEEE
}

\begin{abstract}
In this comment we position the work of [1], [2] in terms of the prior literature and note that many of its results are subsumed by previous papers.
\end{abstract}

Index Terms-Product code, list decoding, iterative decoding, Chase decoding.

$\mathbf{I}$ $\mathrm{N}$ [1], [2] modifications to the decoding of binary block Turbo codes/ Turbo product codes, as described in [3], [4], are considered. Unfortunately, the work ignores much of the existing relevant literature, which subsumes much of their work.

The row and column component codes of the product code $(P C)$ are iteratively decoded as described in [3], [4]. Each soft-input soft-output (SISO) component decoder uses a Chase decoder [5] to generate a list of possible codewords, which is used to calculate the soft output. The modified Chase search described in [1], [2] appears to be the same as the test pattern elimination algorithm presented earlier in [6]. In addition, there are many existing papers which modify the Chase algorithm, including [6], [7], [8], [9], [10].

Various algorithms have been used in place of the Chase algorithm in the SISO decoders of [3], [4]. For example, the the order- $i$ reprocessing algorithm of [11] has been used in [12], [13]. Also Fang et al. developed a different variation in [14]. In addition, the approach of [1], [2], was modified by the authors in [15], [16], both of which should have been referenced in [1]. All these approaches were found to provide significant performance improvements over the Chase-based approach of [3], [4] when the minimum Hamming distance of the component codes was greater than four. The cost is an increase in decoding complexity.

In [3], [4] the extrinsic information passed between decoding stages is scaled by precomputed values to improve performance. In [1], [2] the extrinsic information is scaled by 0.5 and then the hyperbolic tangent of it is taken. In [1], their performance for the $(63,51,5)^{2} \mathrm{PC}$ is compared with that of [3] for the $(64,51,6)^{2}$ PC. Note that these codes have different lengths, rates and minimum Hamming distances and so cannot easily be compared. Performance results for the approach of [3], [4] can be found for the $(63,51,5)^{2} \mathrm{PC}$ in [4].

The approach to scaling the extrinsic information of [1], [2] should also be compared with those in [17], [18]. In [17], [18] adaptive scaling approaches are presented, derived from

Paper approved by S. G .Wilson, the Editor for Coding Theory and Applications of the IEEE Communications Society. Manuscript received March 31, 2004; no revision.

The authors are with the Department of Electrical and Computer Engineering, University of Canterbury, Private Bag 4800, Christchurch, New Zealand (e-mail: \{p.martin, taylor\}@elec.canterbury.ac.nz).

Digital Object Identifier 10.1109/TCOMM.2009.09.040192 log-likelihood ratios. Note that modifications to the approach of [3], including amplitude clipping, are considered in [19].

\section{REFERENCES}

[1] Z. Chi, L. Song, and K. K. Parhi, "On the performance/complexity tradeoff in block turbo decoder design," IEEE Trans. Commun., vol. 52, no. 2, pp. 173-175, Feb. 2004.

[2] — " "A study on the performance, complexity tradeoffs of block turbo decoder design," in Proc. ISCAS, pp. 65-68, May 2001.

[3] R. M. Pyndiah, "Near optimum decoding of product codes: block turbo codes," IEEE Trans. Commun., vol. 46, no. 8, pp. 1003-1010, Aug. 1998.

[4] R. Pyndiah, A. Glavieux, A. Picart, and S. Jacq, "Near optimum decoding of product codes," in Proc. Globecom, pp. 339-343, 1994.

[5] D. Chase, "A class of algorithms for decoding block codes with channel measurement information," IEEE Trans. Inform. Theory, vol. 18, no. 1, pp. 170-182, Jan. 1972.

[6] S. Fragiacomo, C. Matrakidis, and J. O'Reilly, "Novel near maximum likelihood soft decision decoding algorithm for linear block codes," IEE Proc. Commun., vol. 146, no. 5, pp. 265-270, Oct. 1999.

[7] C. M. Hackett, "An efficient algorithm for soft-decision decoding of the $(24,12)$ extended golay code," IEEE Trans. Commun., vol. 29, no. 6, pp. 909-911, June 1981.

[8] N. Y. Yu, Y. Kim, and P. J. Lee, "Iterative decoding of product codes composed of extended hamming codes," in Proc. ISCC, pp. 732-737, 2000.

[9] S. A. Hirst, B. Honary, and G. Markarian, "Fast chase algorithm with an application in turbo decoding," IEEE Trans. Commun., vol. 49, no. 10, pp. 1693-1699, Oct. 2001

[10] N. N. Tendolkar and C. R. P. Hartmann, "Generalization of chase algorithms for soft decision decoding of binary linear codes," IEEE Trans. Inform. Theory, vol. 30, no. 5, pp. 714-721, Sept. 1984.

[11] M. P. C. Fossorier and S. Lin, "Soft-decision decoding of linear block codes based on ordered statistics," IEEE Trans. Inform. Theory, vol. 41, no. 5, pp. 1379-1396, Sept. 1995.

[12] _ _ "Soft-input soft-output decoding of linear block codes based on ordered statistics," in Proc. Globecom, 1998.

[13] P. A. Martin, D. P. Taylor, and M. P. C. Fossorier, "Soft-input soft-output list-based decoding algorithm," in Proc. ISIT, Lausanne, Switzerland, June 2002.

[14] J. Fang, F. Buda, and E. Lemois, "Turbo product code: a well suitable solution to wireless packet transmission for very low error rates," in Proc. $2^{\text {nd }}$ Int. Symp. on Turbo Codes and Related Topics, pp. 101-111, 2000.

[15] Z. Chi and K. K. Parhi, "High speed algorithm and VLSI architecture design for decoding BCH codes," in Proc. ICASSP, pp. 3089-3092, 2002.

[16] —_ "High speed VLSI architecture design for block turbo decoder," in Proc. ISCAS, pp. 901-904, 2002.

[17] A. Picart and R. Pyndiah, "Adapted iterative decoding of product codes," in Proc. Globecom, pp. 2357-2362, 1999.

[18] P. A. Martin and D. P. Taylor, "On adaptive reduced-complexity iterative decoding," in Proc. Globecom, 2000.

[19] Q. Zhang and T. Le-Ngoc, "A decoding algorithm for turbo product codes using optimality test and amplitude clipping," in Proc. Globecom, Nov. 2001. 